\title{
On the influence of reflective boundary conditions on the statistics of Poisson-Kac diffusion processes
}

\author{
Massimiliano Giona*1, Antonio Brasiello ${ }^{2}$, and Silvestro Crescitelli ${ }^{3}$ \\ ${ }^{1}$ Dipartimento di Ingegneria Chimica DICMA Facoltà di Ingegneria, \\ La Sapienza Università di Roma via Eudossiana 18, 00184, Roma, \\ Italy \\ * massimiliano.giona@uniroma1.it \\ ${ }^{2}$ Dipartimento di Ingegneria Industriale Università degli Studi di \\ Salerno via Giovanni Paolo II 132, 84084 Fisciano (SA), Italy \\ ${ }^{3}$ Dipartimento di Ingegneria Chimica, dei Materiali e della \\ Produzione Industriale Università degli Studi di Napoli "Federico II" \\ piazzale Tecchio 80, 80125 Napoli, Italy
}

\begin{abstract}
We analyze the influence of reflective boundary conditions on the statistics of Poisson-Kac diffusion processes, and specifically how they modify the Poissonian switching-time statistics. After addressing simple cases such as diffusion in a channel, and the switching statistics in the presence of a polarization potential, we thoroughly study PoissonKac diffusion in fractal domains. Diffusion in fractal spaces highlights neatly how the modification in the switching-time statistics associated with reflections against a complex and fractal boundary induces new emergent features of Poisson-Kac diffusion leading to a transition from a regular behavior at shorter timescales to emerging anomalous diffusion properties controlled by walk dimensionality of the fractal set.
\end{abstract}

Keywords: Diffusion, Poisson-Kac process, Hyperbolic stochastic models, Transport on Fractals, Emerging statistical properties.

\section{Introduction}

The first, and certainly most intuitive microscopic description of diffusion processes is certainly via particle Brownian motion, dating to a series of papers by A. Einstein [1] and J. Perrin [2. Mathematically, the connection between this archetype of irreversibility (diffusion), and random motion at particle level can be formulated in a rigorous way, starting from the definition of stochastic integration (Ito, Stratonovich, etc.) as a particular form of 
Stieltjes integration, introducing the concept of stochastic differential equations (Langevin equations), and deriving their statistical properties, thus finally obtaining a parabolic equation for the probability density function (the forward Fokker-Planck equation) [3, 4, 5]. The mathematical tool establishing the connection between stochastic dynamics at microscales, and macroscopic diffusion processes is the concept of Wiener processes (also referred to as mathematical Brownian motion). A one-dimensional Wiener process $\mathcal{W}(t)$ is a continuous stochastic process possessing independent increments that are distributed in a Gaussian way (with zero mean and square variance equal to the time elapsed).

Therefore, a diffusion equation of the form

$$
\partial_{t} p(x, t)=D \partial_{x}^{2} p(x, t)
$$

can be viewed as the Eulerian description of a microscopic Lagrangian motion described by the stochastic kinematics

$$
d x(t)=\sqrt{2 D} d w(t),
$$

where $d w(t)$ are the infinitesimal increments of a one-dimensional Wiener process in the time interval $(t, t+d t)$.

Both eqs. (1) and (2) possess fundamental physical limitations. The diffusion equation (11) is characterized by a unbounded propagation velocity that manifestly violates the basic principles of the theory of special relativity. This property is one-to-one with the fractal nature of the graph of a realization $x(t)$ vs $t$ of the stochastic differential equation (2), which, as well known, possesses fractal dimension $3 / 2$ [6].

In order to solve the above mentioned problem, stochastic models possessing finite propagation velocity have been proposed, essentially based on the use of Poisson processes for generating the stochastic perturbations (see Section 21) [7, 8, 9, 10, 11. In the simplest case of one-dimensional spatial problems, the particle moves with constant and bounded velocity, and the stochastic perturbation acts simply by changing the velocity direction (velocity switching), and the statistics of the switching times follows an exponential distribution. The kinematic equations associated with this simple random motion are relativistically consistent and describe a family of stochastic processes that almost everywhere (i.e. apart from the time instants when the switching of the velocity direction occurs) differentiable functions of time.

The basic article addressing this simple stochastic model is due to M. Kac published in 1974 [8], in connection with a stochastic interpretation of the telegraphers' equation. For the sake of historical correctness, the first paper dealing with this class of models is by S. Goldstein [7] that considered similar processes referred to as persistent random walk. Subsequently, Gaveau et al. 
applied this model to derive, via analytic continuation, the one-dimensional Dirac equation of relativistic quantum mechanics [12].

A significant and fertile Literature originates from these observations, using Poisson processes as a model of bounded noise, or as a prototype of colored noise (due to the exponential decay of the correlation function), see [13, 14, 15, 16, 17, 18, 19] just to quote some of the Literature covering different aspects of physical interest.

Henceforth, we will refer to this class of processes as Poisson-Kac processes (see Section 2 for details). In the physical Literature, Poisson-Kac processes are often referred to as dichotomous noise.

While at smaller timescales (smaller than the average time interval between two consecutive velocity switching) the motion is smooth, at larger timescales Poisson-Kac processes retain emergent Brownian properties and, under certain assumptions on the value of the model parameters, they converge to the ordinary Brownian motion case (Kac limit) [8].

In a previous article [20], we have studied the limitations imposed by the wave-like nature of the Poisson-Kac processes on the representation of physically plausible boundary conditions to be applied to the (Eulerian) balance equations for the probability density function (actually, for the probability partial waves, see further Section 2).

In this article, we analyze the impact of reflective boundary conditions, i.e. of impearmability conditions in a closed system, on the statistics of the switching times of Poisson-Kac diffusion processes.

Indeed, due to the wave-like nature of these processes, any form of reflection modifies the exponential switching-time statistics. Reflection conditions act as an active modulation of the Poissonian statistics. Specifically, complex boundary conditions are able to modify the long-term properties of the Poisson-Kac diffusion processes determining anomalous diffusive scalings. This means that the long-term (emerging) fractal properties of the Poisson-Kac processes are altered by complex geometry of the boundary.

The article is organized as follows. Section 2 provides a brief description of Poisson-Kac processes functional to the further developments of the present article, by considering also two-dimensional models that are further analyzed in Section 5. Section 3 introduces the issue of the influence of reflection boundary conditions on the Poissonian statistics in a simple case, namely that of particles in a straight two-dimensional channel (a form of Knudsen diffusion problem [21]). Section 4 addresses another relatively simple, albeit interesting model, namely diffusion in the presence of a potential determining polarization effects at the walls [20. Section 5 addresses the case of fractal boundary conditions by considering Poisson-Kac diffusion on fractal sets (treated in a continuum way, and not merely as a lattice diffusion problem) [22, 23]. This is the first application of Poisson-Kac processes in disordered systems. This model can be also viewed as a stochastic microscopic description of a linear viscoelastic transport problem (transport 
problem with memory) in a fractal medium. However, the main focus in this article is on reflection conditions and how they modify the emerging long-term fractal properties of a Poisson-Kac diffusive trajectory.

\section{Statement of the problem}

Consider the one-dimensional problem of a particle moving in a deterministic potential $U(x)$ under overdamped conditions. If $\eta$ is the friction factor, the particle experiences a deterministic velocity field $v(x)$ given by

$$
v(x)=-\frac{1}{\eta} \partial_{x} U(x),
$$

to which stochastic fluctuations are superimposed. Therefore, the equation of motion can be written as

$$
d x(t)=v(x(t)) d t+\frac{1}{\eta} d F_{\text {stoca }}(t),
$$

where $F_{\text {stoca }}(t)$ is the stochastic force, and $d F_{\text {stoca }}(t)$ its infinitesimal increment. In the case of the classical overdamped Langevin equation, $F_{\text {stoca }}(t)=$ $\eta \sqrt{2 D_{0}} w(t)$ where $w(t)$ is a Wiener process [5]. In the case of the finite propagation velocity model proposed by Kac, $F_{\text {stoca }}(t)$ is the integral over time

$$
F_{\text {stoca }}(t)=b \eta \int_{0}^{t}(-1)^{\chi(\tau)} d \tau,
$$

where $\chi(t)$ is a Poisson process possessing the switching rate $a>0$, i.e., $E[\chi(t) / \chi(0)=0]=a t$, and $b>0$.

The Poisson-Kac equation corresponding to eq. (6) in the presence of a deterministic velocity field $v(x)$ is given by

$$
d x(t)=v(x(t)) d t+b(-1)^{\chi(t)} d t .
$$

Let us indicate with $X(t)$ the stochastic process associated with the equation (6) at time $t$.

Since $(-1)^{\chi(t)}$ can attain solely two values \pm 1 , the statistical description of the process is based on the two partial probability density functions $p^{ \pm}(x, t)$,

$$
p^{ \pm}(x, t) d x=\operatorname{Prob}\left\{X(t) \in(x, x+d x),(-1)^{\chi(t)}= \pm 1\right\},
$$

satisfying the wave equations with recombination (dissipative wave equations)

$$
\begin{aligned}
& \partial_{t} p^{+}(x, t)=-\partial_{x}\left[(v(x)+b) p^{+}(x, t)\right]-a p^{+}(x, t)+a p^{-}(x, t) \\
& \partial_{t} p^{-}(x, t)=-\partial_{x}\left[(v(x)-b) p^{-}(x, t)\right]+a p^{+}(x, t)-a p^{-}(x, t)
\end{aligned}
$$


As these equations correspond to forward and backward propagating waves, the partial probabilities $p^{ \pm}(x, t)$ are also referred to as partial probability waves.

The probability density function $p(x, t)$ for $X(t)$ at time $t$ is the sum of the two partial probability densities $p^{ \pm}(x, t)$

$$
p(x, t)=p^{+}(x, t)+p^{-}(x, t) .
$$

Let us briefly address the properties of the stochastic trajectories associated with eq. (6), and the inclusion of impermeability conditions in finite domains.

First, consider eqs. (6) and (8) in the free space i.e. as the unconstrained propagation along the real line. As $\chi(t)$ is distributed in a Poissonian way, the statistics of the switching times $\tau$, i.e. thta of the time intervals between two consecutive switchings of $(-1)^{\chi(t)}$ is distributed in an exponential way, according to the probability density functions $g_{P}(\tau)$

$$
g_{P}(\tau)=a e^{-a \tau} .
$$

This is the bare Poissonian result. The exponential probability density function for the switchnig times gives rise to the linear recombination terms $\pm\left(-a p^{+}+a p^{-}\right)$entering the balance equations for the partial probabilities (8), determining the exchange from $p^{+}$to $p^{-}$and viceversa per unit time at a constant rate equal to $a$.

Correspondingly, if $a$ and $b$ are bounded, the graph of a generic realization of eq. (6) as a function of time $t$ is an almost everywhere smooth function in all the open intervals between two consecutive switchings. If $t=t^{*}$ is a time instant at which a switching event occurs, solely the derivative of $x(t)$ is discontinuous at $t=t^{*}$, still keeping a bounded left and right derivative.

Let us suppose that eq. (6) describes particle motion in a box, and that $x=0$ and $x=L>0$ are the positions of the box walls, so that particles moving in $(0, L)$ cannot cross them or perform any sort of tunneling at them. Suppose for simplicity that the deterministic contribution $v(x)$ is absent. Impermeability at $x=0, L$ implies total reflections for the partial waves $p^{ \pm}(x, t)$. This means that at $x=0$ the backward propagating waves $p^{-}(x, t)$ is totally reflected, and this determines the boundary condition for $p^{+}(x, t)$, i.e.,

$$
p^{+}(0, t)=p^{-}(0, t) .
$$

In a similar way, the forward wave $p^{+}(x, t)$ is totally reflected at $x=L$, and this induces the complementary boundary condition

$$
p^{-}(L, t)=p^{+}(L, t) .
$$

In terms of stochastic trajectories, reflection at an impermeable wall corresponds to an additional switching of $(-1)^{\chi(t)}$, externally induced by the 
boundaries, that superimposes to the Poissonian exponential statistics. If this occurs at $x=0$, this means that the wall forces the transition of $(-1)^{\chi(t)}$ from -1 to 1 , and similarly at $x=L$, the wall-imposed switching from 1 to -1 occurs.

Consequently, the presence of impermeable boundary conditions provides an active contribution to the statistical properties of the trajectories of the stochastic process (6) determining a modification of the effective switching time statistics $g(\tau)$, which is no longer equal to the bare distribution $g_{P}(\tau)$ expressed by eq. (10). The analysis of $g(\tau)$ and of its consequences on the emerging properties of the solutions of eq. (6) are the main issues of this article.

To conclude this brief introduction to Poisson-Kac processes, let us mention the connection between eq. (6) and the classical Langevin equation driven by Wiener fluctuations. For $a, b \rightarrow \infty$, keeping fixed the ratio

$$
\frac{b^{2}}{2 a}=D
$$

the Poisson-Kac stochastic equation (6) converges to the classical Langevin equation

$$
d x(t)=v(x(t)) d t+\sqrt{2 D} d w(t),
$$

and the probability density function $p(x, t)$ converges to the solution of the Fokker-Planck equation (for mathematical details see also [24])

$$
\partial_{t} p(x, t)=-\partial_{x}[v(x) p(x, t)]=D \partial_{x}^{2} p(x, t) .
$$

This property is referred to as the Kac limit.

\subsection{Two-dimensional Poisson-Kac processes}

Since in Section 5 we consider Poisson-Kac processes in complex two-dimensional structures (fractal sets), it is useful to address here the extension of eq. (6) in higher dimensions, which is an issue scarsely addressed in the physical Literature [25, 26], but very fertile in theoretical probability theory [10, 11].

Although there are very interesting alternative extensions of Poisson-Kac processes in higher dimensions, mainly addressed by Kolesnik and coworkers [10, 11], we focus here on the most natural and straightforward generalization of eq. (6) in two-dimensional domains and in the absence of external potentials or deterministic velocity contributions.

The simplest generalization of eq. (6) in two-dimensional spatial coordinates in the case of pure stochastic motion considers two independent Poisson processes $\chi_{1}(t), \chi_{2}(t)$, each of which acts on distinct spatial coordinates, i.e.,

$$
\begin{aligned}
& d x(t)=b(-1)^{\chi_{1}(t)} d t \\
& d y(t)=b(-1)^{\chi_{2}(t)} d t
\end{aligned}
$$


possessing the same statistical properties, i.e.,

$$
E\left[\chi_{1}(t) / \chi_{1}(0)=0\right]=E\left[\chi_{2}(t) / \chi_{2}(0)=0\right]=a t .
$$

Analogously to the one-dimensional case, the statistical properties of the purely diffusive Poisson-Kac model (16) are fully described by the system of four partial probability density function $p^{( \pm, \pm)}(\mathbf{x}, t)$, here $\mathbf{x}=(x, y)$,

$p^{( \pm, \pm)}(\mathbf{x}, t) d \mathbf{x}=\operatorname{Prob}\left\{\mathbf{X}(t) \in(\mathbf{x}, \mathbf{x}+d \mathbf{x}),(-1)^{\chi_{1}(t)}= \pm 1,(-1)^{\chi_{2}(t)}= \pm 1\right\}$,

where $d \mathbf{x}$ indicates the two-dimensional area element, and $\mathbf{X}(t)=(X(t), Y(t))$ is the two-dimensional stochastic process described by eq. (16) at time $t$.

For the sake of notational simplicity, let us use the notation $p_{1}(\mathbf{x}, t)=$ $p^{(+,+)}(\mathbf{x}, t), p_{2}(\mathbf{x}, t)=p^{(-,+)}(\mathbf{x}, t), p_{3}(\mathbf{x}, t)=p^{(-,-)}(\mathbf{x}, t), p_{4}(\mathbf{x}, t)=p^{(+,-)}(\mathbf{x}, t)$, so that the overall probability density function for $\mathbf{X}(t))$ is the sum of the $p_{h}$ 's,

$$
p(\mathbf{x}, t)=\sum_{h=1}^{4} p_{h}(\mathbf{x}, t) .
$$

It can be easily recognized that each $p_{h}(\mathbf{x}, t)$ describes the statistics of a particle ensemble moving with constant velocity $b \boldsymbol{\beta}_{h}$, where

$$
\boldsymbol{\beta}_{h}=\left(\cos \left(\frac{2(h-1) \pi+\pi}{4}\right), \sin \left(\frac{2(h-1) \pi+\pi}{4}\right)\right),
$$

$h=1, \ldots, 4$. As it regards the recombination dynamics, consider for example $p_{1}=p^{(+,+)}$, and use the notation $( \pm, \pm)$to indicate $\left((-1)^{\chi_{1}(t)}= \pm 1,(-1)^{\chi_{2}(t)}= \pm 1\right)$. Considering the statistics of an ensemble of particles, the switching in the unit time from $(+,+)$ to $(-,+)$ and $(+,-)$ occurs with rate $a$ The reverse processes possess obviously the same rate $a$. Consequently, the balance equation for $p_{1}(\mathbf{x}, t)$ is the Eulerian continuity equation for a particle ensemble moving with constant velocity $b \boldsymbol{\beta}_{1}$ which accounts for the above discussed recombination dynamics amongs partial waves,

$$
\partial_{t} p_{1}(\mathbf{x}, t)=-b \nabla \cdot\left(\boldsymbol{\beta}_{1} p_{1}(\mathbf{x}, t)\right)-2 a p_{1}(\mathbf{x}, t)+a\left(p_{2}(\mathbf{x}, t)+p_{4}(\mathbf{x}, t)\right) .
$$

where $\nabla$ indicates the nabla operator with respect to the spatial coordinates $\mathbf{x}$. In a similar way, the balance equations for the other partial waves follow

$$
\begin{gathered}
\partial_{t} p_{2}(\mathbf{x}, t)=-b \nabla \cdot\left(\boldsymbol{\beta}_{2} p_{2}(\mathbf{x}, t)\right)-2 a p_{2}(\mathbf{x}, t)+a\left(p_{1}(\mathbf{x}, t)+p_{3}(\mathbf{x}, t)\right) \\
\partial_{t} p_{3}(\mathbf{x}, t)=-b \nabla \cdot\left(\boldsymbol{\beta}_{3} p_{3}(\mathbf{x}, t)\right)-2 a p_{3}(\mathbf{x}, t)+a\left(p_{2}(\mathbf{x}, t)+p_{4}(\mathbf{x}, t)\right) \\
\partial_{t} p_{4}(\mathbf{x}, t)=-b \nabla \cdot\left(\boldsymbol{\beta}_{4} p_{4}(\mathbf{x}, t)\right)-2 a p_{4}(\mathbf{x}, t)+a\left(p_{1}(\mathbf{x}, t)+p_{3}(\mathbf{x}, t)\right) .
\end{gathered}
$$

Gathering eqs. (21)-(22) and summing over the index " $h$ " of the partial waves, the balance equation for the overall probability density function follows

$$
\partial_{t} p(\mathbf{x}, t)=-\nabla \cdot \mathbf{J}(\mathbf{x}, t)
$$


where $\mathbf{J}(\mathbf{x}, t)$ is the probability flux originating from the stochastic bivariate Poissonian perturbation

$$
\mathbf{J}(\mathbf{x}, t)=b \sum_{h=1}^{4} \boldsymbol{\beta}_{h} p_{h}(\mathbf{x}, t) .
$$

From eqs. (21)-(24), it is straightforward to obtain the constitutive equation for the probability flux, multiplying each evolution equation for $p_{h}(\mathbf{x}, t)$ by $b \boldsymbol{\beta}_{h}$ and summing over the index " $h$ ",

$$
\partial_{t} \mathbf{J}(\mathbf{x}, t)=-b^{2} \nabla \cdot\left(\sum_{h=1}^{4} \boldsymbol{\beta}_{h} \boldsymbol{\beta}_{h} p_{h}(\mathbf{x}, t)\right)-2 a \mathbf{J}(\mathbf{x}, t) .
$$

Several observations follows from the system of equations (23) $-(\underline{25})$ :

- the statistical description of the process corresponds to that of a linear viscoelastic material with memory, as the constitutive equation for $\mathbf{J}(\mathbf{x}, t)$ depends on the first-order time derivative of the flux itself;

- this representation is however irreducible in terms of $p(\mathbf{x}, t)$ and $\mathbf{J}(\mathbf{x}, t)$, as the correct description of the process involves the full system of partial probability waves $\left\{p_{h}(\mathbf{x}, t)\right\}_{h=1}^{4}$;

- although there are some analogy between eq. (25) and the Cattaneo transport equation with memory in higher dimensions [27, 28], the functional form of the constitutive equation (25) marks the fundamental difference between the two models. For finite values of $a$ and $b$, it is impossible to express the time-derivative of the probability flux $\mathbf{J}(\mathbf{x}, t)$ solely as a function of $\mathbf{J}(\mathbf{x}, t)$ itself and of the overall probability density function $p(\mathbf{x}, t)$ : all the systems of $\left\{p_{h}(\mathbf{x}, t)\right\}_{h=1}^{4}$ should be considered. If the first term at the r.h.s. of eq. (25) is approximated by a term proportional to the gradient of $p(\mathbf{x}, t)$, as in the two-dimensional Cattaneo model [27, 28], the stochastic interpretation of the resulting constitutive equation is completely lost, and this is the fundamental reason while the higher-dimensional Cattaneo model does not satisfy the requirement of positivity [20];

- the classical diffusion equation follows from (22)-(25) in the Kac limit.

To prove the last observation, consider the limit for arbitrarily large $a$ and $b(a, b \rightarrow \infty)$, keeping fixed the ratio $b^{2} / a$ to a constant value. If the switching rate tends to infinity, the recombination process between partial waves becomes infinitely fast, and the structure of the partial waves "thermalizes" at any $\mathbf{x}$, meaning that all the $p_{h}(\mathbf{x}, t)$ collapse into a unique 
function that is just $p(\mathbf{x}, t) / 4$. Enforcing this property into eq. (25) the constitutive equation for the probability flux becomes

$$
\mathbf{J}(\mathbf{x}, t)=-\frac{b^{2}}{2 a} \nabla \cdot(\mathbf{B} p)-\frac{1}{2 a} \partial_{t} \mathbf{J}(\mathbf{x}, t),
$$

where $\mathbf{B}$ is the diadic tensor

$$
\mathbf{B}=\frac{1}{4} \sum_{h=1}^{4} \boldsymbol{\beta}_{h} \boldsymbol{\beta}_{h} .
$$

Because of the structure of the normalized velocities $\boldsymbol{\beta}_{h}$ defined by eq. (20), this tensor is isotropic and equal to the identity tensor, $\mathbf{B}=\mathbf{I}$. In the Kac limit, the second term at the right hand side of eq. (26) vanishes and the constitutive equation reduces to the Fickian form, $\mathbf{J}(\mathbf{x}, t)=-D \nabla p(\mathbf{x}, t)$, where the diffusivity is given by eq. (13).

Finally, let us discuss the role of impermeability conditions. Consider for simplicity the problem of Poisson-Kac diffusion inside a square, $(x, y) \in$ $(0, L) \times(0, L)$. Because of the symmetries of the normalized velocities $\boldsymbol{\beta}_{h}$, each reflection at the box walls, placed at $x=0, L, y=0, L$ corresponds to an externally forced switching either of $(-1)^{\chi_{1}(t)}$ (for the reflections at $x=0, L)$ or of $(-1)^{\chi_{2}(t)}$ (for the reflections at $\left.y=0, L\right)$. The boundary conditions for the partial waves at the walls can be obtained either by enforcing that the normal component of the probability flux should vanish at the box walls or by expressing total reflection in terms of the partial waves as it result from the switching of one of the $(-1)^{\chi_{h}(t)}, h=1,2$. For example, consider the collisions with the boundary at $x=0$. In this case $(-1)^{\chi_{1}(t)}$ switches from the value $(-1)^{\chi_{1}\left(t_{-}\right)}=-1$ to the value $(-1)^{\chi_{1}\left(t_{+}\right)}=1$, and consequently the boundary conditions become

$$
p_{1}(0, y, t)=p_{3}(0, y, t), \quad p_{4}(0, y, t)=p_{2}(0, y, t),
$$

meaning that the two backward waves (along the $x$ coordinate) $p_{3}$, and $p_{2}$ are reflected back into $p_{1}$ and $p_{4}$, respectively. Analogous expressions can be derived for the other wall collisions.

\section{The simplest case: Knudsen effect}

The simplest physical situation where the effects of impermeable walls controls the statistics of Poisson-Kac diffusion processes is represented by particle motion in straight channels under creeping conditions, so that the deterministic velocity fields acts only along the axial direction. Consider a two-dimensional model letting $x$ be the axial, and $y$ the transverse coordinates. 
Particle motion is described (using nondimensional variables) by the system of stochastic equations

$$
\begin{aligned}
d x(t) & =v(y(t)) d t+b(-1)^{\chi_{1}(t)} d t \\
d y(t) & =b(-1)^{\chi_{2}(t)} d t
\end{aligned}
$$

where $y \in(0,1)$, equipped with reflection boundary conditions at $y=0,1$ which represent channel walls. As the transverse motion is completely decoupled from the axial one, we can consider the second equation (29) as a stand-alone problem. The Poisson process $\chi_{2}(t)$ is characterized by a switching rate $a$, and we assume $b$ and $a$ to be related by the Kac condition (13), i.e. $b^{2}=2 D a$, where $D$ is the nondimensional diffusion coefficient. Therefore, instead of $a$ and $b$ we can use $b$ and $D$ or $a$ and $D$ to define completely the characteristic of the stochastic perturbation that controls transverse particle motion. ppp Figure 1 depicts the time series of the consecutive switching intervals $\tau_{n}$ vs the numeral order $n$. The most evident effect of impermeable boundaries is that the switching intervals $\tau_{n}$ are upper bounded, as the maximum attainable value of the switching time is given by $\tau_{\max }=L / b$, where $L$ is the transverse width of the channel, and $b$ the characteristic velocity of the stochastic perturbation. In the present case, $L=1$, and $b=\sqrt{2 D a}$, so that

$$
\tau_{\max }=\frac{1}{\sqrt{2 D a}} .
$$

For $D=a=1, \tau_{\max }=1 / \sqrt{2}$, corresponding to the horizontal dashed line in figure 1 .

This result follows from the elementary observation that the longest switching time is reached by a particle hitting consecutively the two walls without switching the velocity direction due to the Poissonian statistics. Consequently, the probability density function $g(\tau)$ for the switching times admits a compact support represented by the interval $\left[0, \tau_{\max }\right]$, while the bare Poissonian $g_{P}(\tau)$ does not.

This effect can be referred to as the Knudsen effect on the Poissonian statistics induced by wall reflection, to mark the analogy with the phenomenon of Knudsen diffusion for dilute gases in narrow pores where the collision with the pore walls dominate the diffusive transport process.

Figure 2 depicts the behavior of the switching time pdf $g(\tau)$ obtained from the stochastic simulation of eq. (29). The pdf $g(\tau)$ can be approximated by the superposition of a smooth exponential distribution defined in $\left[0, \tau_{\max }\right]$ possessing a decay exponent $a_{e} \neq a$, different from the Poissonian switching rate $a$, and of an impulsive contribution centered at $\tau_{\max }$, i.e.,

$$
g(\tau)=A e^{-a_{e} \tau}+B \delta\left(\tau-\tau_{\max }\right),
$$

where $A$ and $B$ are positive constants, such that $A\left(1-e^{-a_{e} \tau_{\max }}\right) / a_{e}+B=1$. 


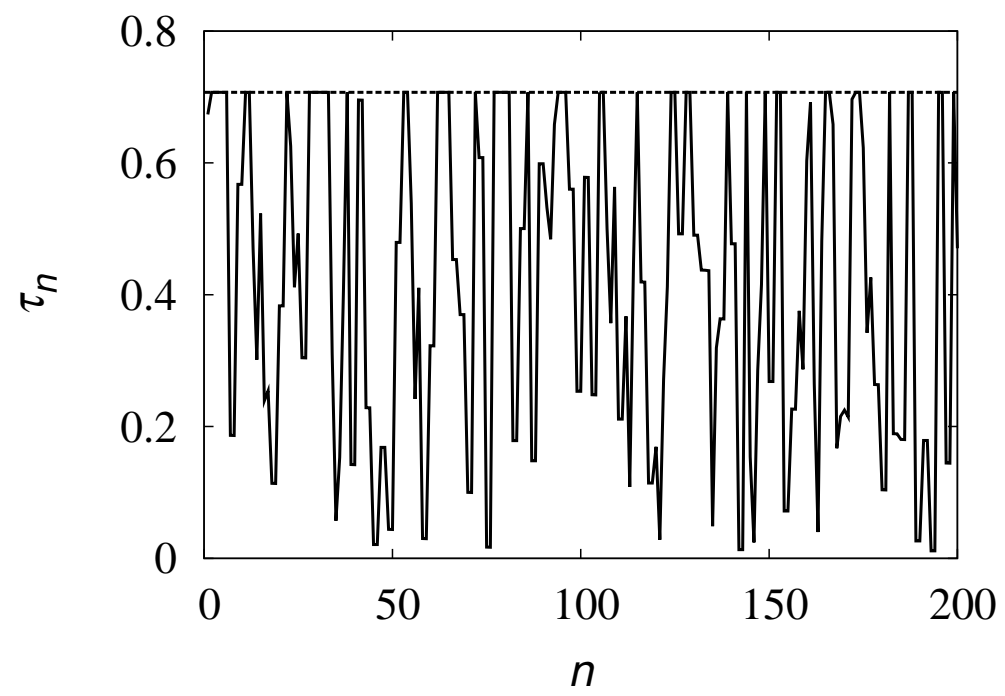

Figure 1: Time series of the switching intervals $\tau_{n}$ vs $n$ for a Poisson-Kac diffusive trajectory at $D=1, a=1$.

The effective rate depends on $a$ and $D$, and its rescaled behavior, i.e., $\left(a_{e}-a\right) / a$, is depicted in figure 3 as a function of $a$ for different values of $D$. As can be observed, the relative deviation from the Poissonian rate $a$ is more pronounced as $D$ and $a$ increase.

It is possible to obtain a quantification of the distortion effects induced by wall reflections using simple arguments. Consider the fraction $\phi_{\text {wall }}$ of the number of switching events associated with wall collisions with respect to the overall number of switchings. A lower bound for $\phi_{\text {wall }}$ can be obtained by considering that this fraction should necessarily be greater than the Poissonian probability of having switching times greater than $\tau_{\max }$, i.e.,

$$
\phi_{\mathrm{wall}} \geq \int_{\tau_{\max }}^{\infty} g_{P}(\tau) d \tau=e^{-a \tau_{\max }}=e^{-\sqrt{a / 2 D}}=\phi_{0}(a, D) .
$$

Figure 4 depicts the behavior of $\phi_{\text {wall }}$ and of its lower-bound estimate $\phi_{0}(a, D)$ as a function of $a$ for two values of $D$. The estimate $\phi_{0}(a, D)$ approaches better the value $\phi_{\text {wall }}$ at lower values of $a$ and higher values of $D$.

\section{Polarization model}

As a second example, consider the one-dimensional model in the presence of a deterministic biasing field,

$$
d x(t)=v(x(t)) d t+b(-1)^{\chi(t)} d t
$$




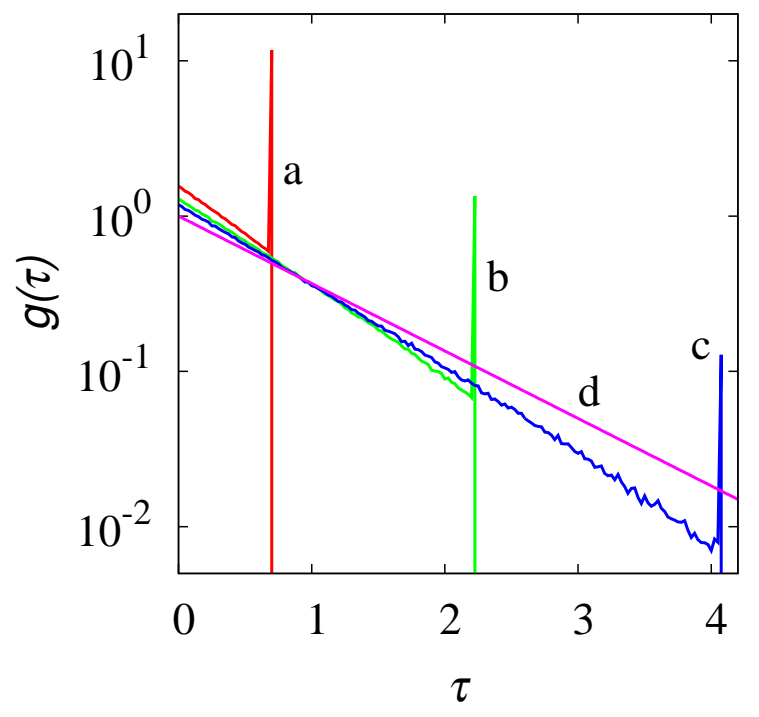

Figure 2: Switching time probability density function $g(\tau)$ vs $\tau$ for the onedimensional Kac diffusion process on the unit interval for $a=1$ at different values of the diffusivity $D$. Line (a): $D=1$, line (b): $D=0.1$, line (c) $D=0.03$. Line $(\mathrm{d})$ corresponds to the pure Poisson statistics $g_{P}(\tau)=a e^{-a \tau}$ in the absence of boundary condition effects.

defined for $x \in[0,1]$, equipped with impermeability conditions at $x=0,1$. The velocity vield $v(x)$ is given by

$$
v(x)=-v_{m} \sin \left(\frac{3 \pi x}{2}\right),
$$

and $v_{m}=\gamma b$, with $\gamma \in(0,1)$, and $a$ and $b$ are such that $D=1$. This case has been analyzed elsewhere [20], as a model for describing polarization effects within the Poisson-Kac paradigm, arising as a consequence of a nonvanishing value of the deterministic velocity field at one of the boundary points $(x=1)$, determining the formation of a polarization layer in the neighbourhood of $x=1$.

In this article, we use this model to investigate better the conditions to be imposed on the stochastic dynamics (33) in the presence of a nonvanishing deterministic bias at the boundary.

First, consider the partial waves associated with eq. (33), i.e. its Eulerian description. The overall probability density flux $J_{\text {tot }}(x, t)$ is the sum of the convective (deterministic) and stochastic contribution,

$$
J_{\text {tot }}(x, t)=v(x)\left[p^{+}(x, t)+p^{-}(x, t)\right]+b\left[p^{+}(x, t)-p^{-}(x, t)\right] .
$$




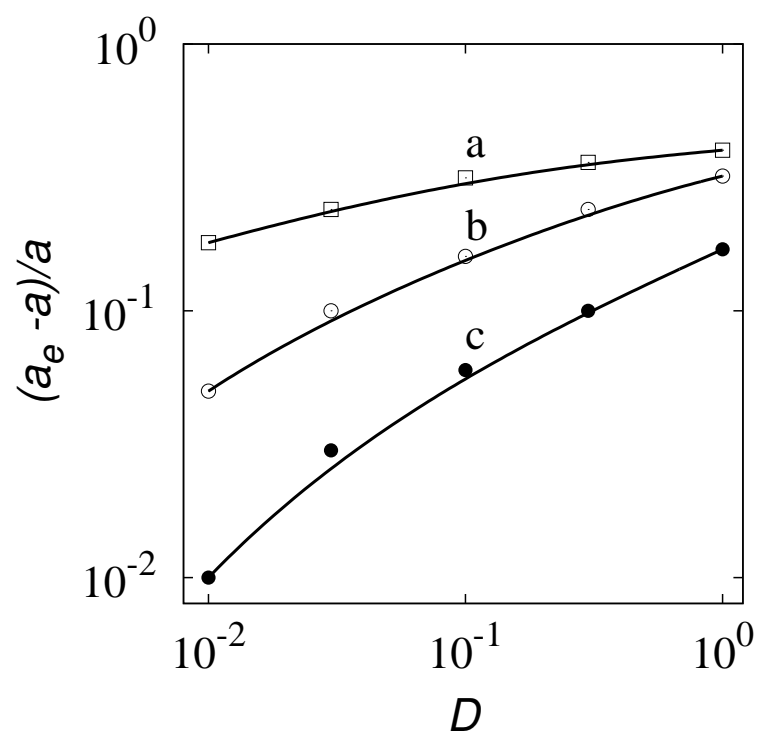

Figure 3: Rescaled effective switching rate $\left(a_{e}-a\right) / a$ vs diffusivity $D$ at three different values of $a$. Line (a) and ( $\square$ ): $a=1$, line (b) and (o): $a=0.1$, line (c) and $(\bullet): a=0.01$.

Since $v(0)=0$, the zero-flux condition at $x=0$ simply becomes

$$
p^{+}(0, t)=p^{-}(0, t) .
$$

This is the total reflection condition. At $x=1, v(1)=\gamma b$, and the zero-flux boundary condition becomes in terms of partial waves

$$
p^{-}(1, t)=\frac{1+\gamma}{1-\gamma} p^{+}(1, t) .
$$

Eq. (37) is valid for $\gamma<1$. The extension to $\gamma>1$ is discussed in [20], and therefore it is not repeated here.

For the stochastic differential equation (33) the conditions at the boundary are simply reflection conditions, corresponding to the mirror simmetric reflection of the particle position at $x=0,1$, and to the switching of $(-1)^{\chi(t)} \mapsto-(-1)^{\chi(t)}$ of the Poissonian perturbation.

In order to check the validity of these conditions, we compare the stationary solutions $p_{*}^{ \pm}(x)$ of the associated partial wave model (8) with $v(x)$ given by eq. (34), in the presence of the boundary conditions (36)-(37), and the overall stationary probability density function $p_{*}(x)=p_{*}^{+}(x)+p_{*}^{-}(x)$, with the corresponding stationary distributions obtained by integrating the stochastic equation of motion (33) with an integration step $h_{t}=10^{-4}$ for an ensemble of $N=10^{7}$ particle in which the above mentioned simple reflection 


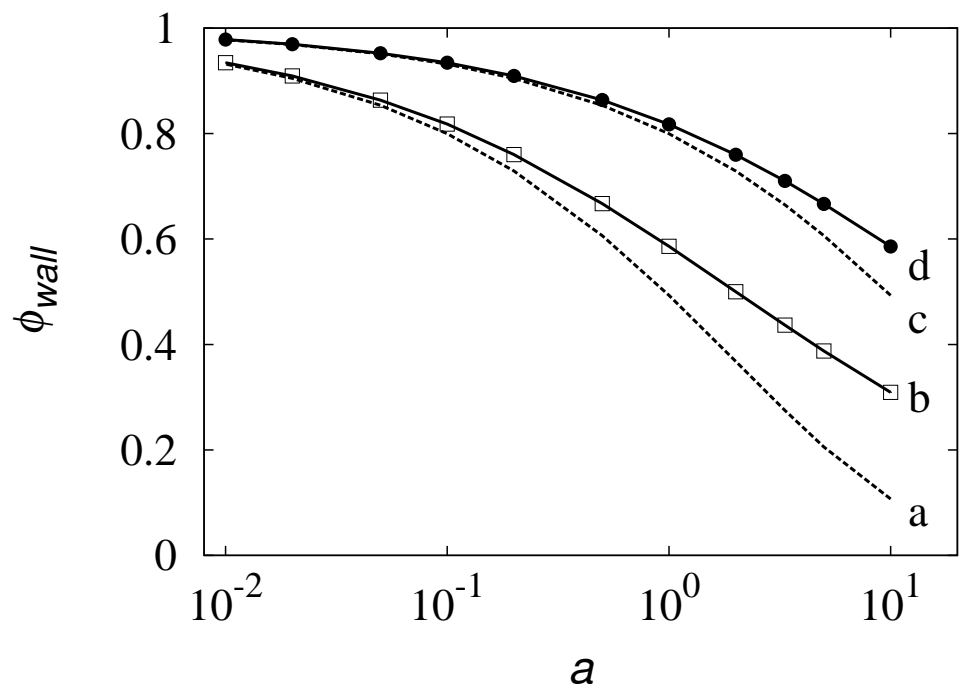

Figure 4: $\phi_{\text {wall }}$ vs the switching rate $a$ for two values of $D$. Symbols $(\square, \bullet)$ represent simulation results, dashed lines the lower bound eq. (32). Lines (a) and (b) and symbols ( $\square$ ): $D=1$, lines (c) and (d) and symbols $(\bullet)$ : $D=10$.

conditions have been used at the boundaries. This comparison is depicted in figure 5 and is fully satisfactory.

In point of fact, a more stringent test on the influence of the boundary conditions (especially at $x=1$ ) is based on the analysis of the ratio $p_{*}^{-}(x) / p_{*}^{+}(x)$, that, because of eqs. (36) and (37) should attains the values 1 at $x=0$, and $(1+\gamma) /(1-\gamma)$ at $x=1$. Figure 6 shows the estimate of this ratio obtained from the stochastic evolution of the particle ensemble compared with the corresponding quantity obtained from the partial wave model at different values of $\gamma$.

The dashed horizontal lines correspond to the limit values $(1+\gamma) /(1-\gamma)$ at $x=1$ for the values of $\gamma$ considered. Also in this more severe test, the stochastic model equipped with the simple reflecting boundary conditions provide excellent quantitative agreement with the Eulerian results.

The switching time distributions $g(\tau)$ obtained from stochastic simulations is depicted in figure 7 for several values of $\gamma$. Compared with the corresponding results obtained for the simple Knudsen model described in Section 3 , there are qualitative analogies and differences. The analogies are: (i) the compactness of the support of $g(\tau)$, and (ii) the occurrence of an impusive contribution centered at $\tau_{\max }$. The differences are: (i) a significant modification in the low- $\tau$ region induced by the polarization effects of the 


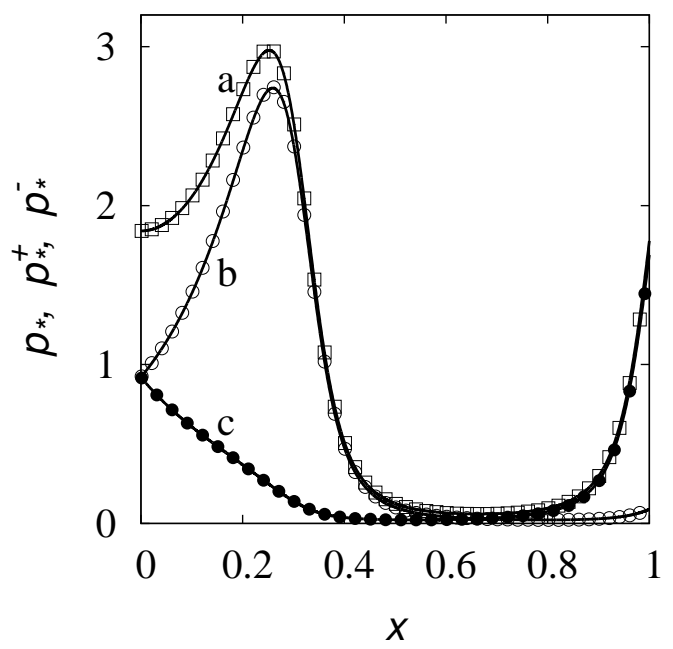

Figure 5: Stationary probability distribution $p_{*}(x)$ (lines (a) and ( $\square$ )), and stationary partial waves $p_{*}^{+}(x)$ lines $(\mathrm{b})$ and $\left.(\circ)\right), p_{*}^{-}(x)$ (lines (a) and $\left.(\bullet)\right)$ for the polarization model (33) at $D=1, a=5, \gamma=0.9$. Solid lines represent the results of the integration of the partial wave equations, symbols the results of stochastic simulations of eq. (33).

velocity field near the boundaries, and (ii) the exponential "backbone" of this distribution, that apart for impulsive and nearly impulsive contribution possesses an exponential decay equal to $a$, i.e., equal to the bare Poissonian switching rate (compare the decay of the Poissonian statistics, line (e) with mean local decay of the other lines (a) to (d)).

\section{$5 \quad$ Kac diffusion on fractals}

As a final example, consider diffusion in complex geometries such as in twodimensional connected sets possessing fractal (Hausdorff) dimension $1<$ $d_{f}<2$. It is known that Brownian diffusion in fractal media possesses anomalous properties [22, 23], typically expressed by the power-law scaling of the mean square displacement $\left\langle r^{2}(t)\right\rangle$ of Brownian particles

$$
\left\langle r^{2}(t)\right\rangle \sim t^{2 / d_{w}}
$$

where $d_{w}>2$ is the walk dimension $\left(d_{w}=2\right.$ in Euclidean media, giving rise to the classical Einsteinian linear relation of $\left\langle r^{2}(t)\right\rangle \sim t$ ).

Most of the existing numerical results on diffusion in fractal media refer to lattice simulations, where Brownian particles move in a random and uncorrelated way from a site of the fractal set to one of its neighbouring sites belonging to the set itself. 


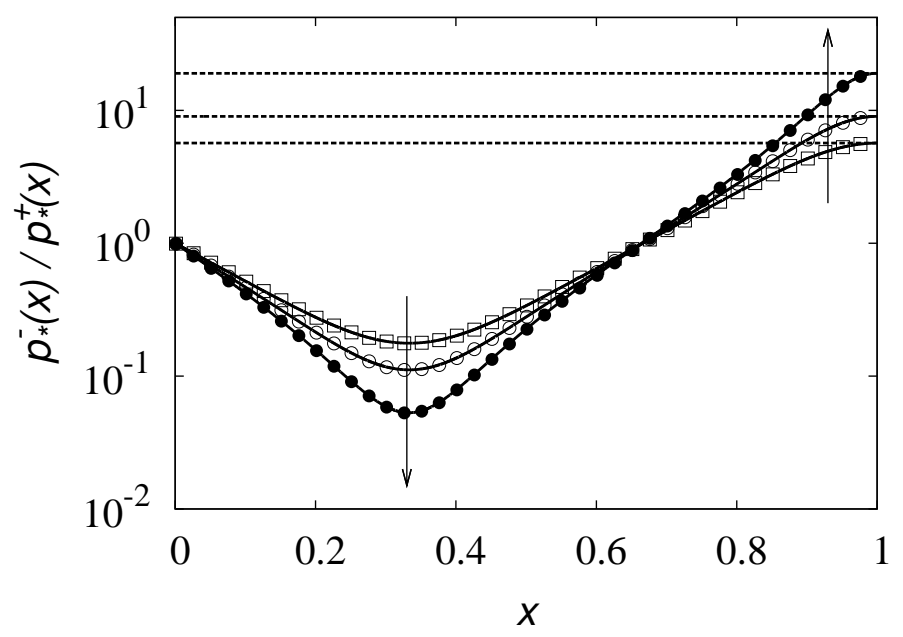

Figure 6: Ratio $p_{*}^{-}(x) / p_{*}^{+}(x)$ under stationary conditions at $D=1, a=5$. Solid lines represent the results of the integration of the partial wave equations, symbols the results of stochastic simulations of eq. (33). Arrows indicate increasing values of $\gamma=0.7,0.8,0.9$. Dashed horizontal lines corresponds to the limit value at $x=1,(1+\gamma) /(1-\gamma)$.

In order to give concrete examples, consider the two fractal structures depicted in figure 8: the Sierpinski carpet, and a loopless deterministic fractal, henceforth referred to as the deterministic cross fractal. Their fractal dimensions are $d_{f}=\log 8 / \log 3 \simeq 1.893$ (for the Sierpinski carpet), and $d_{f}=\log 5 / \log 3 \simeq 1.465$ for the deterministic cross fractal. Anomalous diffusion properties on the Sierpinski carpets have been addressed in [29, 30].

Figure 9 depicts the behavior of the mean square displacement of classical Brownian particles obtained from discrete lattice simulations. The simulations refer to a prefractal approximation of the structures at the 7-th iteration of the costruction process, that corresponds to lattices possessing $3^{7}=2187$ lattice size per Cartesian coordinate. In point of fact, figure 8 depicts just these prefractal approximations, as can be noticed by the labels of $x$ and $y$ coordinates.

From the scaling of the mean square displacement (line a) we obtain the numerical value $2 / d_{w}=0.94$ for the Sierpinski carpet (no analytic results are available for the Sierpinski carpet, which is an infinitely ramified structure not easily amenable to exact real-space renormalization). In the case of the deterministic cross fractal, the scaling theory of loopless fractals, based on the concept of chemical distance, predicts a simple relation between $d_{w}$ and $d_{f}$, namely [22, 23]

$$
d_{w}=d_{f}+1
$$




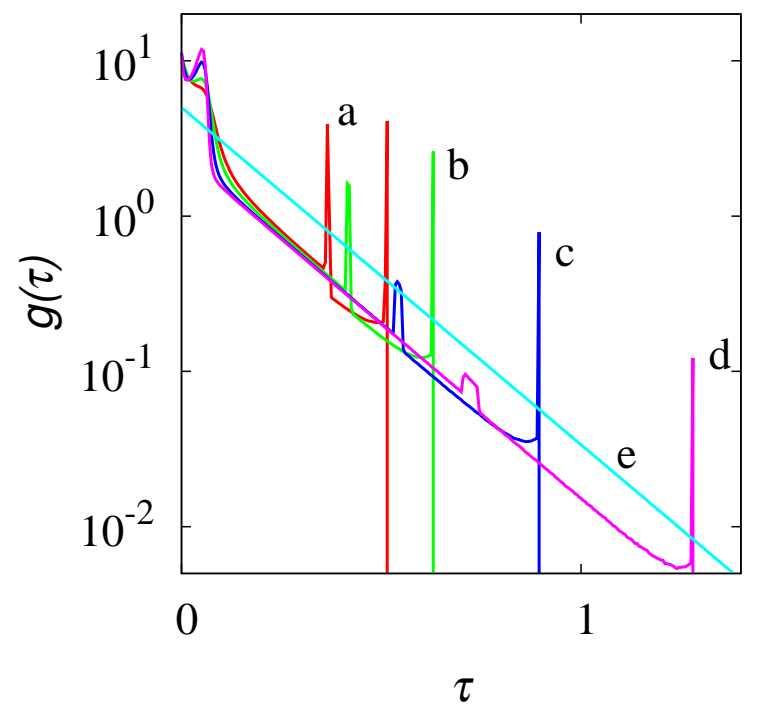

Figure 7: Switching time probability density function $g(\tau)$ vs $\tau$ for the polarization model (33)-(34) at $a=5$. Line (a): $\gamma=0.7$, line (b): $\gamma=0.8$, line (c): $\gamma=0.9$, line (d) $\gamma=0.95$. Line (e) represents the pure Poissonian distribution $g_{P}(\tau)=a e^{-a \tau}$.

and the numerical results perfectly agree with the scaling $\left\langle r^{2}(t)\right\rangle \sim t^{2 /\left(d_{f}+1\right)}$ (line b).

Next, consider Poisson-Kac diffusion in these sets. The mathematical setting of the problem has been developed in paragraph 2.1, both as it regards the stochastic equations of motion and their statistical (Eulerian) description in terms of partial waves. In the numerical simulations of eq. (16) we consider that the unit site of the prefractal approximation possesses a unit linear length. Consequently, the unit square is the building block of the fractal structure, that at iteration $n=7$ extends over a length of $L=3^{7}=2187$. Using eq. (16), and applying total reflection conditions whenever a particle hits the boundary (which implies also the switching of $(-1)^{\chi_{1}(t)}$ or $(-1)^{\chi_{2}(t)}$ depending on the which boundary is involved), it is possible to perform an off-lattice (continuous) simulation of the Poisson-Kac diffusion process. Let $D=1$ be the nondimensional diffusion coefficient. Figure 10 panels (a) to (c) depict a portion of an orbit of a Poisson-Kac particle diffusing inside the Sierpinski carpet at different values of $a$. An analogous picture for the deterministic cross fractal is shown in figure 11 . 

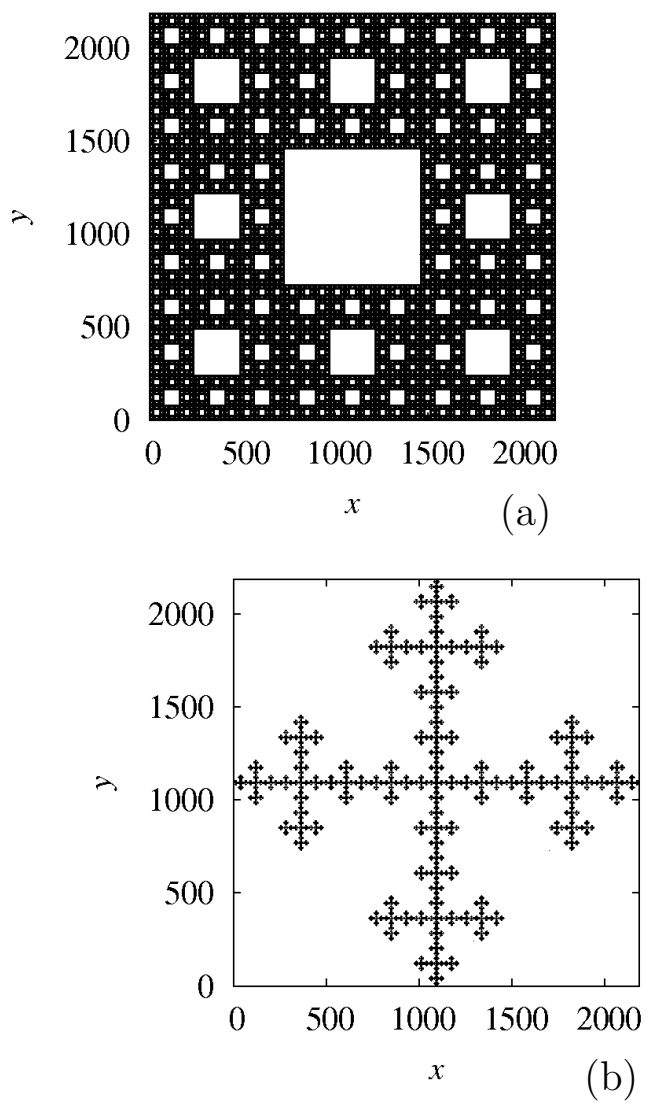

Figure 8: Panel (a): Sierpinski carpet. Panel (b): deterministic cross fractal.

At small values of $a$, figure 10 panel (b) and figure 11, (keeping $D$ fixed and consequently, varying $b$ according to the Kac condition eq. (13i)), the orbits are significantly different from that of classical Brownian motion, as the switching of the Poissonian processes is controlled by collisions with the boundary of the fractal structure. As a increases (see panel (b) and (c) in figure 10), particle orbits resemble more closely that of classical Brownian particles. Figure 12 depict the mean square displacement of PoissonKac particles in the two fractal structures considered at several values of the switching rate $a$. The mean square displacement $\left\langle r^{2}(t)\right\rangle$ possesses two asymptotic scalings: at short time scales the mean square displacement is ballistic, and this is to be expected from the wave nature of the stochastic process, as for $t<<\min \{1 / b, 1 / a\}$ particles move in straight lines. In the long-term limit, the mean square displacement possesses exactly the same anomalous behavior characterizing classical Brownian particles.

Anomalous diffusion effects, i.e. the occurrence of a value of $d_{w}>2$, 


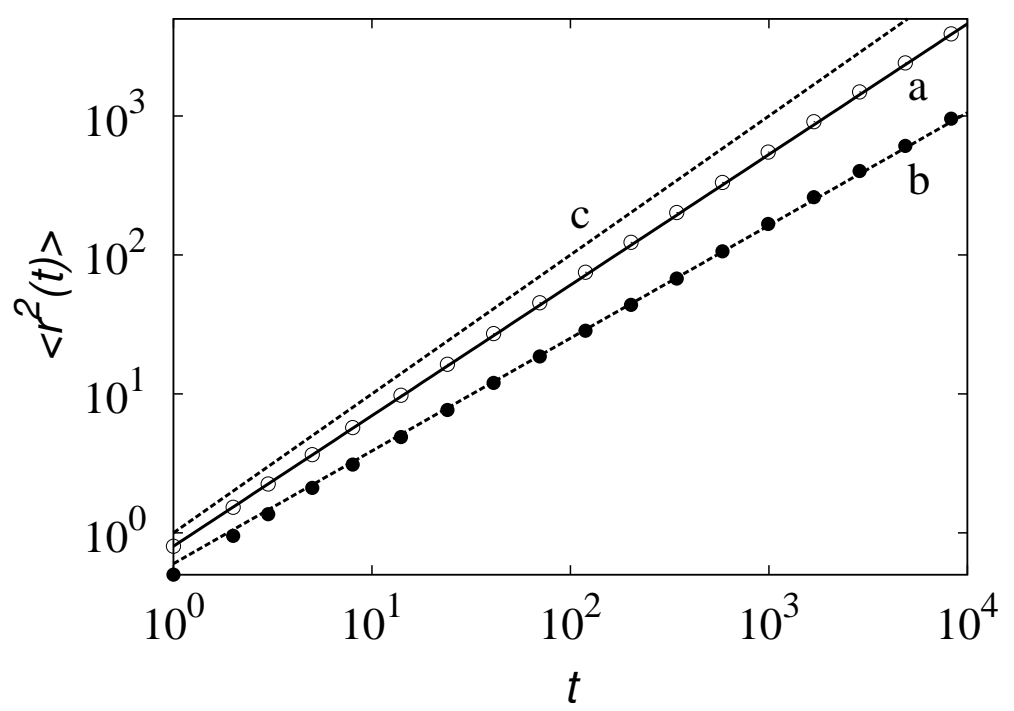

Figure 9: Mean square displacement $\left\langle r^{2}(t)\right\rangle$ vs $t$ for classical Brownian lattice diffusion on the fractal structures considered. Symbols are the results on lattice random simulations: (o): Sierpinski carpet, $(\bullet)$ : deterministic cross fractal. Lines (a) and (b) represent the scaling $\left\langle r^{2}(t)\right\rangle \sim t^{2 / d_{w}}$, with $2 / d_{w}=$ 0.94 (line (a)), and $2 / d_{w}=2 /\left(d_{f}+1\right), d_{f}=\log 5 / \log 3$ (line (b)). Line (c) represents $\left\langle r^{2}(t)\right\rangle \sim t$.

are in the Poisson-Kac case the emerging feature of the collisions with the boundary of the fractal structure, and therefore it is to be expected that the switching time statistics becomes significantly modified. As the process is two-dimensional, we consider separately the statistics $g_{\alpha}(\tau), \alpha=1,2$ for the switchings of the two Poissonian processes. The hit of the boundaries orthogonal to the $x$-axis determines a switching of $(-1)^{\chi_{1}(t)}$, and the reflection onto a boundary orthogonal to the $y$-axis determines a switching of $(-1)^{\chi_{2}(t)}$.

Figure 13 and 14 depict the behavior of $g_{\alpha}(\tau) \alpha=1,2$ obtained from stochastic simulations. The two density functions $g_{1}(\tau), g_{2}(\tau)$ are equal and differ from the pure Poissonian statistics $g_{P}(\tau)$. Nevertheless, their behavior is well approximated by an exponential decay with an effective decay rate $a_{e}$ different from $a$.

A coarse information of the role of the boundary collisions can be obtained by considering the ratio $\phi_{\text {coll }}$ of the overall number of collisions with the walls to the number of switchings in the velocity determined by the Poissonian statistics. The behavior of $\phi_{\text {coll }}$ vs $a$ is depicted in figure 15 at $D=1$. It can be observed that this ratio follows a power law behavior 

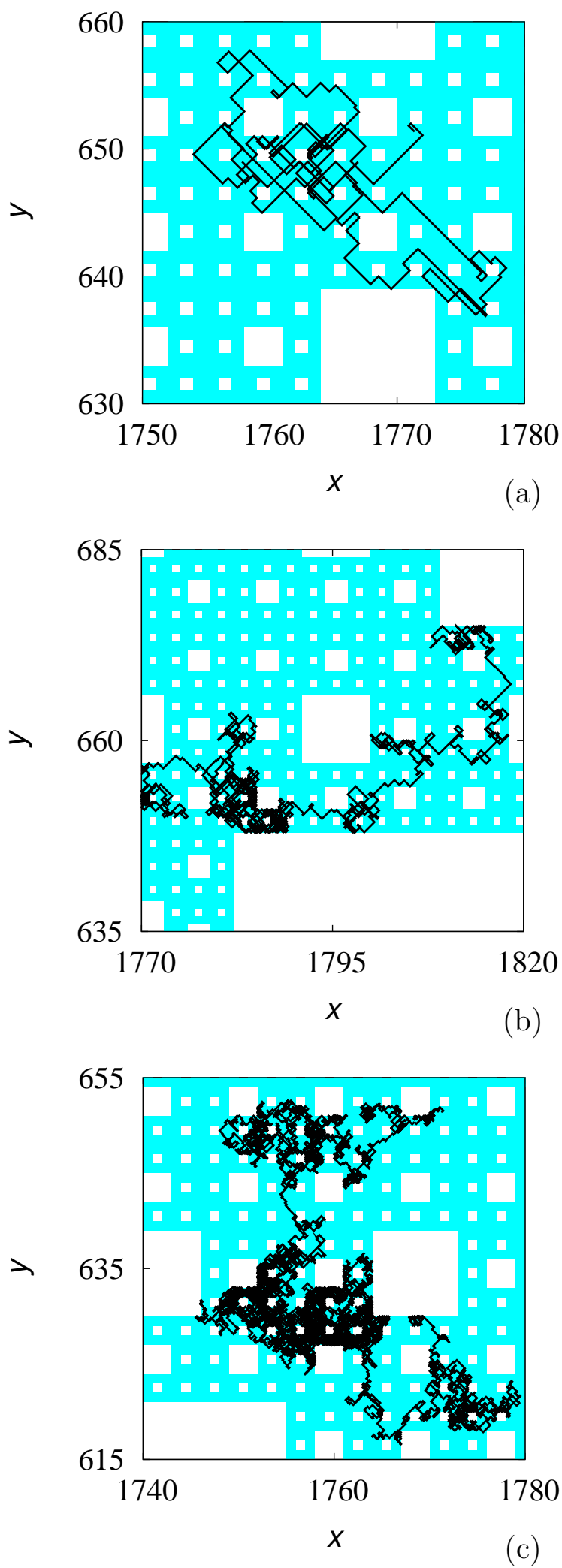

20

Figure 10: Realizations of diffusive Kac orbits on the Sierpinski carpet at $D=1$. Panel (a): $a=0.1$, panel (b): $a=1$, panel (c) $a=10$. 


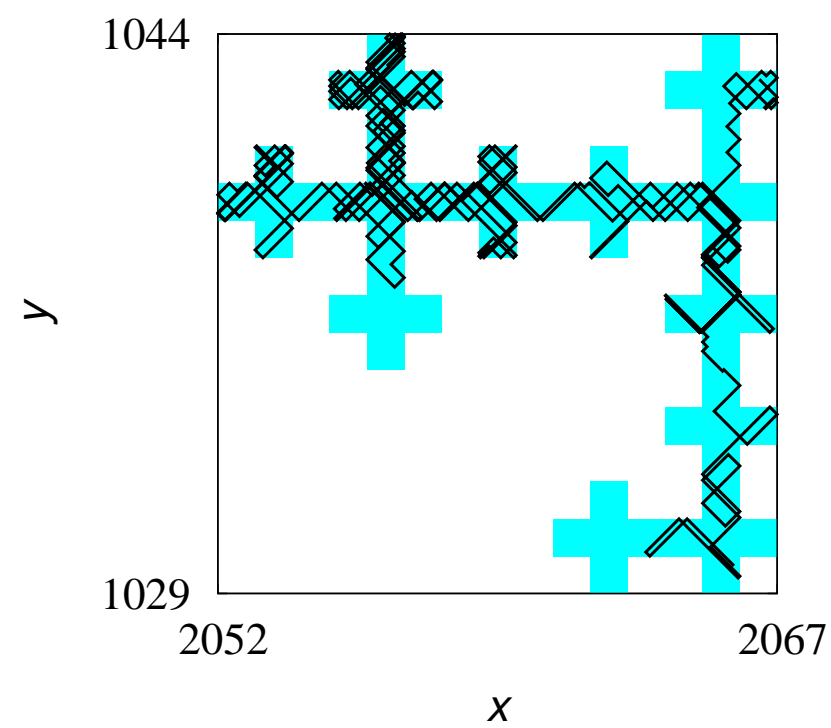

Figure 11: Realization of a diffusive Kac orbit on the deterministic cross fractal at $D=1$ and $a=0.1$.

$\phi_{\text {coll }}(a) \sim a^{-\zeta}$, where the exponent $\zeta$ attains the value (from the best fit of simulation data): $\zeta=0.72$ for the Sierpinski carpet, $\zeta=0.79$ for the deterministic cross fractal. This power law scaling is nor related in a simple way to the characteristic geometric $\left(d_{f}\right)$ and dynamic $\left(d_{w}\right)$ exponents of the medium. We leave a more careful theoretical analysis on the properties of this scaling law, and of its framing within the theory of transport in fractal media to future investigations.

Let us analyze in more detail the trajectories of Poisson-Kac particles on fractals. By definition, the trajectories of the realizations of Poisson-Kac processes are with probability 1 almost eveywhere smooth curves. Discontinuities in the derivatives arise as a consequence of the "internal" Poissonian switching occurring in average at a characteristic time $\tau_{c}=1 / a$, and as a result of the "external" reflections with the boundaries. Since the unit building block of the fractal structure possesses unitary characteristic length, the average time between collisions with the boundary is $\tau_{w}=1 / b=1 / \sqrt{2 D a}$.

For time scales much smaller than the minimum between $\tau_{c}$ and $\tau_{w}$, the trajectories are simply straight lines. Figure 16 depicts two examples of particle trajectory (actually its $x$-component) at two different values of $a$. The higher $a$ is, the smaller is the characteristic timescale at which the realization of Poisson-Kac processes attain asymptotic fractal properties.

The role of wall collisions that superimpose to the intrinsic Poissonian switchings is therefore to induce in the long-term behavior of particle trajec- 


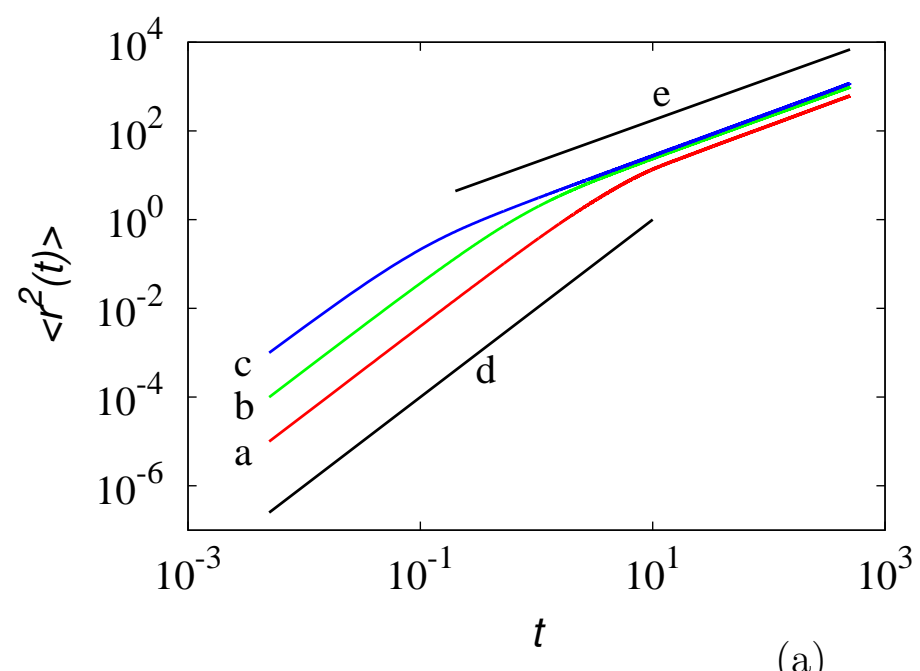

(a)

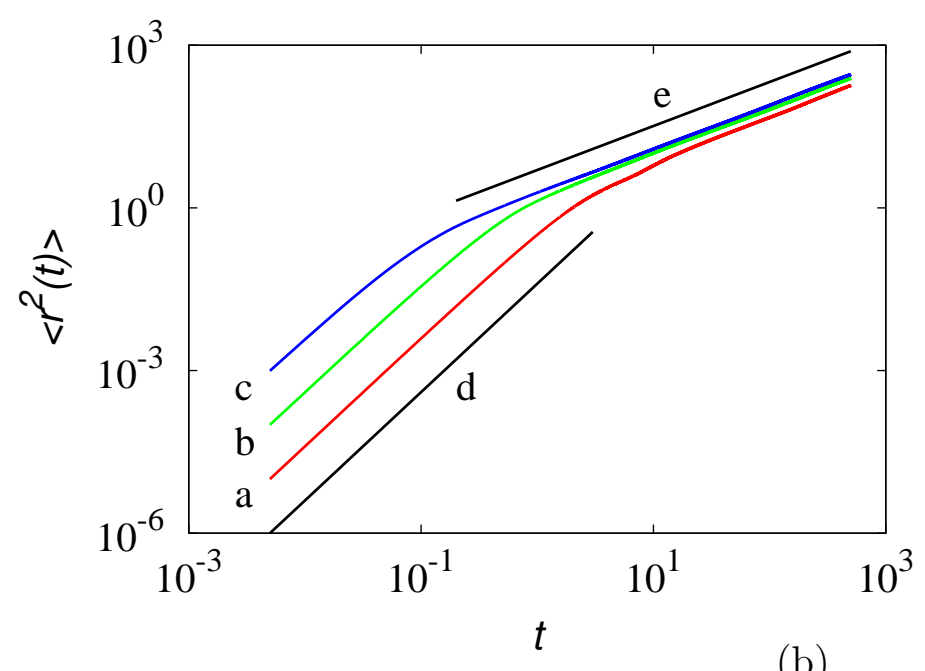

(b)

Figure 12: Mean square displacement $\left\langle r^{2}(t)\right\rangle$ vs time $t$ for Kac diffusion on Sierpinski carpet (panel a) and on the deterministic cross fractal (panel b) at $D=1$. Lines (a) to (c) refer to different values of $a=0.1$ (lines a), $a=1$ (lines b), $a=10$ (lines c). Lines (d) correspond to the scaling $\left\langle r^{2}(t)\right\rangle \sim t^{2}$. Lines (e) to the anomalous diffusion scaling $\left\langle r^{2}(t)\right\rangle \sim t^{2 / d_{w}}$, where $2 / d_{w}=0.94$ (panel a), and $2 / d_{w}=0.8114$ (panel b).

tories a fractal behavior characterized by a fractal dimension different from $3 / 2$, that corresponds to the classical Brownian motion in Euclidean media.

Let $d_{T}$ be the fractal dimension of Poisson-Kac trajectories in the longterm regime, and $L\left(\Delta t ; t_{\max }\right)$ the length of a finite portion of the trajectory in the interval $t \in\left[0, t_{\max }\right)$ estimated using a temporal yardstick $\Delta t$. Hence- 

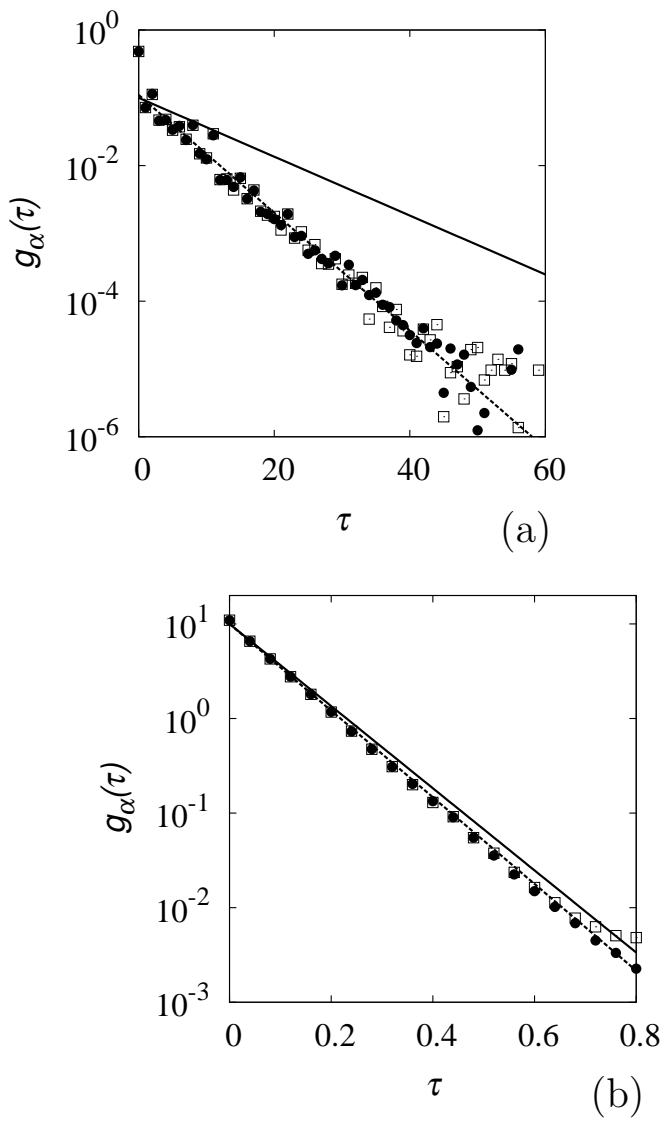

Figure 13: Switching time probability density functions $g_{\alpha}(\tau), \alpha=1,2$, vs $\tau$ for diffusion on the Sierpinski carpet at $D=1$. Panel (a): $a=0.1$, panel (b): $a=10$. Symbols $(\square)$ refer to $g_{1}(\tau)$, symbols $(\bullet)$ to $g_{2}(\tau)$. The solid line is the bare Poisson statistics $g_{\alpha}(\tau)=a e^{-a t}$, the dashed line is the exponential fit of the data.

forth, for notational simplicity, we drop the reference to $t_{\max }$ and write simply $L(\Delta t)$.

It follows from the above qualitative analysis that

$$
L(\Delta t) \sim \begin{cases}\text { constant } & \Delta t<\Delta t^{*} \\ \Delta t^{1-d_{T}} & \Delta t \gg \Delta t^{*},\end{cases}
$$

where the crossover time $\Delta t^{*}$ depends on $a$. The fractal dimension $d_{T}$ is related to the Hurst exponent of the graph of the trajectories by the relation 31]

$$
d_{T}=2-H,
$$




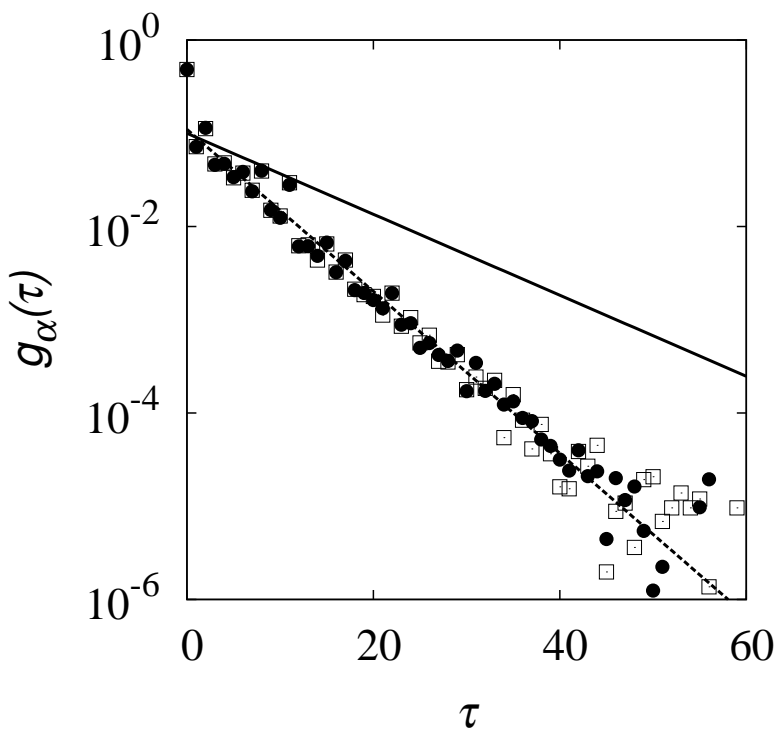

Figure 14: Switching time probability density functions $g_{\alpha}(\tau), \alpha=1,2$, vs $\tau$ for diffusion on the deterministic cross fractal at $D=1, a=1$. Symbols $(\square)$ refer to $g_{1}(\tau)$, symbols $(\bullet)$ to $g_{2}(\tau)$. The solid line is the bare Poisson statistics $g_{\alpha}(\tau)=a e^{-a t}$, the dashed line is the exponential fit of the data.

and, in turn, it is related to the walk dimension $d_{w}$ by $H=1 / d_{w}$, so that

$$
d_{T}=2-\frac{1}{d_{w}} .
$$

Consequently, the length-yardstick analysis, typical of the geometric characterization of fractal curves [31], provides an alternative way to estimate $d_{w}$ from a single realization of the process.

Figure 17 depicts the graphs of $L(\Delta t)$ vs $\Delta t$ for several Poisson-Kac processes on the Siepinski carpet and on the deterministic cross fractal at different values of $a$. The realization of the process has been obtained by integrating eq. (16) with a time step $h_{t}=2 \times 10^{-3}$ up to $t_{\max }=4 \times 10^{2}$, starting from a point inside the fractal structure chosen at random.

The data depicted in figure 17 agree with the scaling (40). For small $\Delta t<\Delta t^{*}$, where $\Delta t^{*}$ decreases with $a, L(\Delta t)$ saturate, due to the rectifiable nature of the trajectories. For $\Delta t>\Delta t^{*}$ the fractal scaling emerges as a long-term property of the process. From eqs. (40)-(42) it follows that

$$
L(\Delta t) \sim \Delta t^{1 / d_{w}-1}, \quad \Delta t \gg \Delta t^{*},
$$

and the value of $d_{w}$ estimated from the long-term fractal scaling of $L(\Delta t)$ using eq. (43) perfectly agrees quantitatively with the value of the walk dimension obtained from the analysis of particle mean square displacement. 


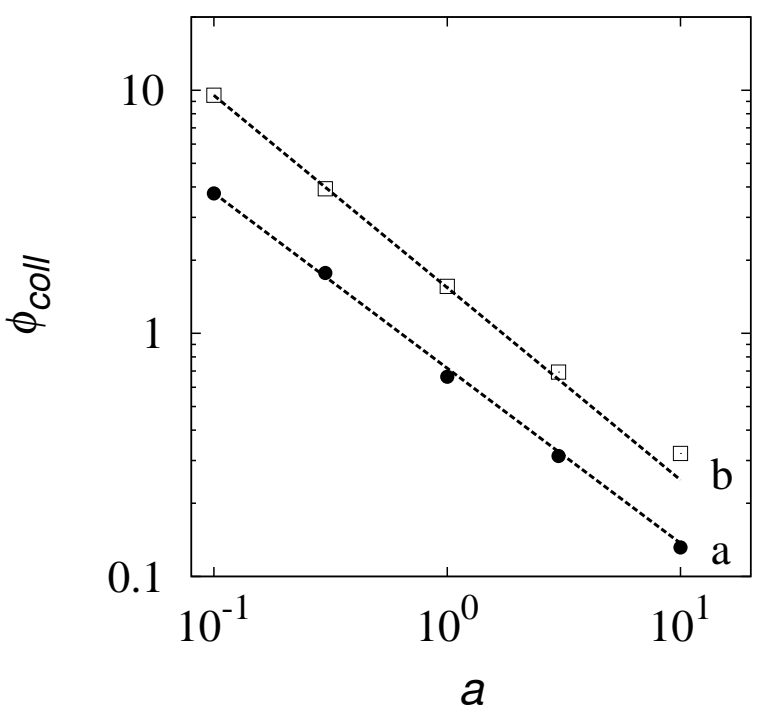

Figure 15: $\phi_{\text {coll }}$ vs $a$ for Kac diffusion on fractals at $D=1$. Solid lines represent the scaling $\phi_{\text {coll }}(a) \sim a^{-\zeta}$. Line (a) and $(\bullet)$ refer to the Sierpinski carpet $(\zeta=0.72)$, line (b) and $(\square)$ to the deterministic cross fractal $(\zeta=$ $0.79)$.

As expected, the fractal scaling appears more neatly (i.e., over a larger range of $\Delta t$ ) if one considers higher values of $a$ (curves (c) in figure 17), since, for fixed $D$, the characteristic stochastic velocity $b=\sqrt{2 D a}$ is higher and the collisions with the fractal boundary, determining the occurrence of the emergent fractal behavior of the trajectories, stabilize the process at smaller $\Delta t$.

\section{Concluding remarks}

The statistics of Poisson-Kac processes in closed systems is profoundly affected by reflection conditions at the boundaries. This effect influences either the statistics of the switching times and the emerging long-term properties of the transport process. The study of Poisson-Kac diffusion on fractals clearly shows that the anomalies in diffusion occur as emerging properties of a local, almost everywhere differentiable, stochastic motion at microscales, deriving from the complex collision process occurring at the boundary of a fractal set, and determining the transition from a ballistic motion to anomalous Einstein scaling characterized by a walk dimension greater than 2 .

The crossover in the mean square displacement has its dynamic counterpart in the properties of the trajectories deriving from the analysis of 


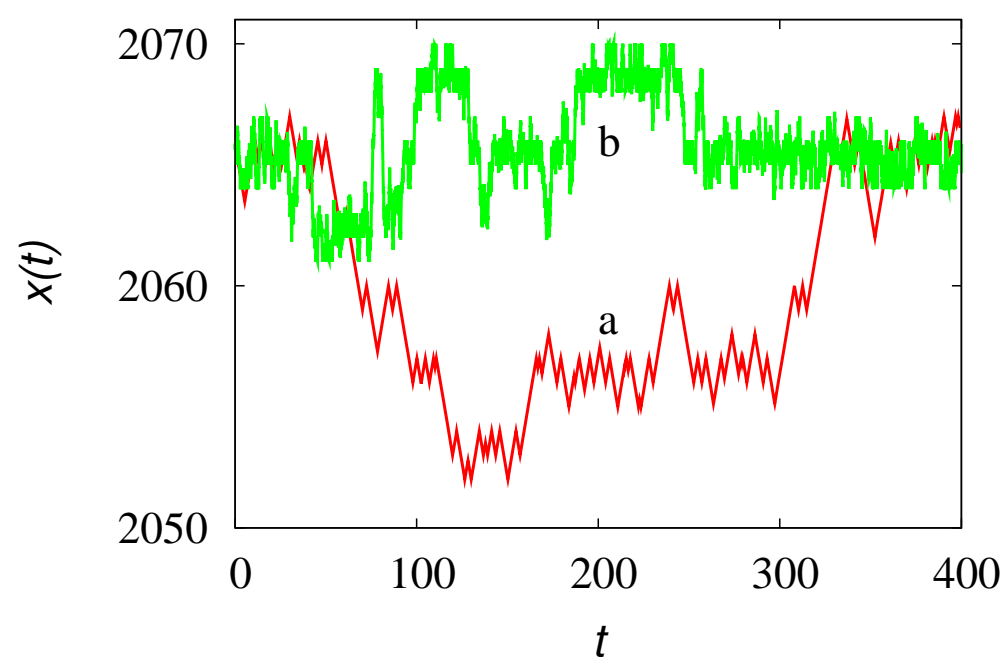

Figure 16: $x$-component $x(t)$ of a diffusive Kac trajectory vs $t$ on the deterministic cross fractal at $D=1$. Line (a) refers to $a=0.1$, line (b) to $a=10$.

the scaling of the length $L(\Delta t)$ vs the timescale $\Delta t$. The trajectories of Poisson-Kac diffusive particles admit a transition from a local smooth behavior at shorter timescales to the emerging fractal properties characterized by a trajectory fractal dimension $d_{T}=2-1 / d_{w}$.

Two observations deserve further attention. Poisson-Kac processes addressed in Section 5 are indeed useful tools to investigate, using off-lattice algorithms, complex transport processes in fractal and disordered media, either in the case of pure diffusive motion (as in the present work) or including the effects of deterministic velocity fields and potentials. At relatively small values of $a$ and $b$, they represent the stochastic transport of a kind of a viscoelastic phase with memory, and memory effects influence the short-term behavior. Conversely the long-term, long-distance properties of Poisson-Kac processes are those of classical Brownian motions.

However, Poisson-Kac processes do not share with the classical Brownian motion all the conceptual problems associated with an infinite propagation velocity, and therefore they can be consistently applied also to relativistic problems.

The second observation is purely technical. In order to highlight the long-term fractality of Poisson-Kac trajectories emerging from locally differentiable stochastic motion we have used the length/time-interval scaling (figure 17), out of which the Hölder exponent $H$ of the stochastic trajec- 

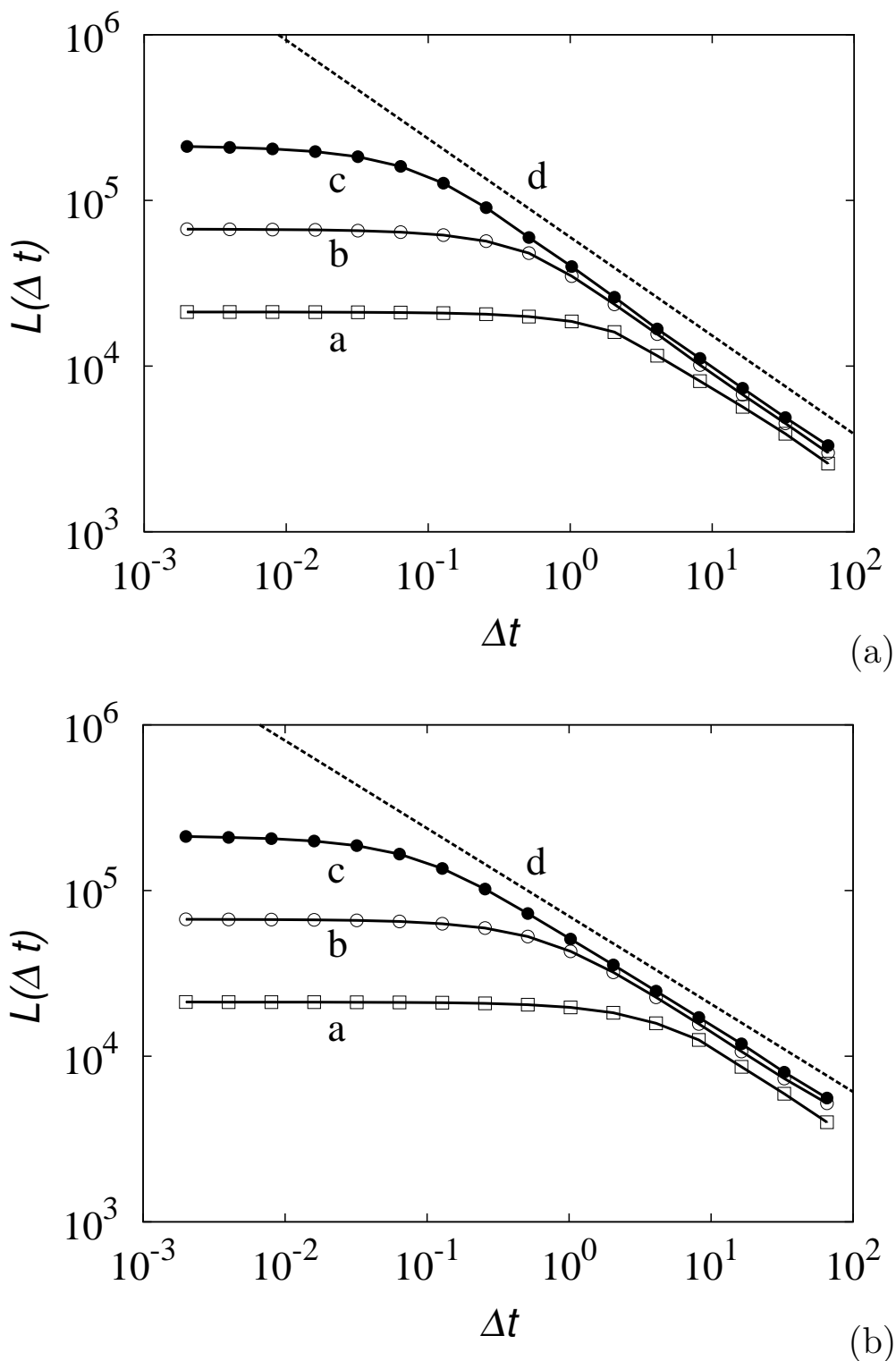

Figure 17: Scaling of the length $L(\Delta t)$ vs the temporal yardstick size $\Delta t$ on a diffusive Kac trajectory at $D=1$. Panel (a) refers to the deterministic cross fractal, panel (b) to the Sierpinski carpet. Lines (a) and ( $\square$ ) correspond to $a=0.1$, lines (b) and (o) to $a=1$, lines (c) and $(\bullet)$ to $a=10$. Lines (d) corresponds to the theoretical prediction $L(\Delta t) \sim \Delta t^{1 / d_{w}-1}$, where $d_{w}=$ $\log 5 / \log 3+1$ in panel (a), and $d_{w}=2.4648$ in panel (b).

tories can be estimated. Since $H$ is related to the walk dimension $d_{w}$, the scaling of $L(\Delta t)$ vs $\Delta t$ provides a reliable, accurate and efficient way to ana- 
lyze anomalous transport properties in fractal media, alternative to the more classical analysis of the scaling of the mean square displacement $\left\langle r^{2}(t)\right\rangle$, just using a single particle trajectory.

\section{References}

\section{References}

[1] A. Einstein, Investigations on the Theory of the Brownian Movement (Dover Publ., New York, 1956).

[2] J. Perrin, Brownian Movement and Molecular Reality (Taylor \& Francis, London, 1910).

[3] C. W. Gardiner, Handbook of stochastic methods for physics, chemistry and the natural sciences (Springer-Verlag, Berlin, 1994).

[4] H. Risken Fokker-Planck Equation (Springer-Verlag, Berlin, 1984).

[5] H. C. Öttinger, Stochastic Processes in Polymeric Fluids (SpringerVerlag, Berlin, 1996).

[6] K. Falconer, Fractal Geometry. mathematical Foundations and Applications (J. Wiley, Chichister, 2014).

[7] S. Goldstein, Quart. J. Mech. Appl. Math. IV (1951), 129.

[8] M. Kac, Rocky Mount. J. Math. 4 (1974), 497.

[9] P. Rosenau, Phys. Rev. E 48 (1993), R655

[10] A.D. Kolesnik, J. Stat. Phys. 131 (2008), 1039.

[11] A. Kolesnik and A. F. Turbin, Stoch. Proc. Appl. 75 (1998), 67.

[12] B. Gaveau, T. Jacobson, M. Kac, L.S. Schulman, Phys. Rev. Lett. 53 (1984), 419.

[13] W. Horsthemke and R. Lefever, Noise-Induced Transitions (SpringerVerlag, Berlin, 1984).

[14] J. M. Sancho, J. Math. Phys. 25 (1984), 354.

[15] J. Masoliver and G.H. Weiss, Eur. J. Phys. 17 (1996), 190.

[16] G. H. Weiss, Physica A 311 (2002) 381.

[17] P. Hänggi and P. Jung, Adv. Chem. Phys. 89 (1995), 239. 
[18] I. Bena, Int. J. Mod. Phys. 20 (2006), 2825.

[19] F. Laio, L. Ridolfi, P. D’Odorico, Phys. Rev. E 78 (2008), 031137.

[20] A. Brasiello, S. Crescitelli, M. Giona, "One-dimensional hyperbolic transport: positivity and admissible boundary conditions derived from the wave formulation", submitted to Physica A, 2015.

[21] R. E. Cunningham and R. J. J. Williams, Diffusion in Gases and Porous Media (Plenum Press, New York, 1980).

[22] S. Havlin and D. Ben-Avraham, Adv. Phys. 36 (1987) 695.

[23] D. Ben-Avraham and S. Havlin, Diffusion and reactions in fractals and disordered systems, (Cambridge University Press, Cambridge, 2000).

[24] A. Janssen, J. Theor. Prob. 3, (1990), 349.

[25] M. Boguná, J. M. Porrà and J. Masoliver, Phys. Rev. E 59 (1999), 6517.

[26] A. V. Plyukhin, Phys. Rev. E 81 (2010), 021113.

[27] C. Cattaneo, Atti del Seminario Mat. Fis. Univ. Modena 3 (1948) 3; C.R. Acad. Sci. 247 (1958) 431.

[28] D. Jou, J. Casas-Vazquez and G. Lebon, Extended Irreversible Thermodynamics, (Springer, New York, 2010).

[29] R. Dasgupta, T.K. Ballabh and T. Tarafdar, Phys. Lett. A, 187 (1994), 71.

[30] R. Dasgupta, T.K. Ballabh and T. Tarafdar, J. Phys. A 32 (1999), 6503.

[31] C. Tricot, Curves and fractal dimension, (Springer, New York, 1995). 